\title{
ENHANCEMENT OF SOLUBILITY AND BIOAVAILABILITY OF BCS CLASS-II AMBRISENTAN: IN VITRO, IN VIVO AND EX VIVO ANALYSIS
}

\author{
RAHUL RADKE*, NEETESH K. JAIN
}

Faculty of Pharmacy, Oriental University, Indore, M. P. India

*Email: rahul.radke@rediffmail.com

Received: 06 Oct 2021, Revised and Accepted: 17 Nov 2021

\begin{abstract}
Objective: The aim of this investigation was to enhance the solubility and bioavailability of the BCS class II poorly water-soluble drug ambrisentan by solid dispersion (SD) techniques using Gelucire 50/13 as a hydrophilic carrier.

Methods: Solid dispersion of ambrisentan was prepared by kneading method using different dug: carrier ratios. Prepared SD was characterized for solubility, drug content, percentage yield, in vitro dissolution, ex vivo permeation and bioavailability. Solid-state characterization was performed by differential scanning calorimetry (DSC), X-ray diffraction (XRD) and scanning electron microscopy (SEM).

Results: All the SDs formulations showed increase in drug solubility and dissolution when compared with its pure form. Aqueous solubility of the drug was found to be increased 8.23 fold in SD. DSC study showed that endothermic peak of the drug was disappeared in spectra of SD, confirming its amorphous conversion, XRD study revealed the reduction to almost absence of specific high-intensity peaks of drug which confirmed the reduction of crysatallinity of ambrisentan in SD. SEM of optimized SD formulation demonstrates the complete encapsulation and solubilization drug. In vitro dissolution study showed that optimized SD formulation (ASD4) gives the faster drug release of $101.5 \%$ in 60 min, as compare to its pure form and other SD formulations.
\end{abstract}

Conclusion: Solid dispersion ASD4 prepared with 1:4 drug to carrier ratio showed the highest drug solubility and in vitro dissolution. The ex vivo and in vivo studies performed on optimized formulation ASD4 showed enhancement in drug permeability and bioavailability in Gelucire 50/13 based SD formulation.

Keywords: Ambrisentan, Gelucire 50/13, Bioavailability, Solid dispersion, Kneading technique etc

(C) 2022 The Authors. Published by Innovare Academic Sciences Pvt Ltd. This is an open access article under the CC BY license (https://creativecommons.org/licenses/by/4.0/) DOI: https://dx.doi.org/10.22159/ijap.2022v14i1.43347. Journal homepage: https://innovareacademics.in/journals/index.php/ijap

\section{INTRODUCTION}

Absorption of a drug from oral route majorly depends up on the dissolution of the drug from the formulations into GI fluids followed by its permeation. Bioavailability of drugs from oral route depends on their solubility as well as permeability. In order to achieved desired pharmacological response, the solubility of the drug is one of the important parameter [1,2]. Low bioavailability of the drugs are generally associated with poor aqueous solubility which is turn required the higher dose and repeated administration of drug. Bioavailability of poorly water-soluble drugs can be increased by increasing its aqueous solubility and dissolution rate [3]. Solid dispersion is one of the widely utilized technology in the improvement of the solubility, dissolution and bioavailability of poorly water-soluble drugs $[4,5]$.

Solid dispersion prepared with novel surfactants and selfemulsifiers-based carrier is found to advantageous in the improvement drug solubility and dissolution rate. Gelucire 50/13 is a mixtures of monoesters, diesters, and triesters of glycerol and monoesters and diesters of polyethylene glycols was a specially designed polymer for the enhancement of poorly soluble drugs [6]. SD prepared with Gelucire $50 / 13$ is supposed to reduce the crystallinity and convert the drug in to amorphous form so as to enhance the bioavailability of low water-soluble drugs [7].

Ambrisentan is endothelin receptor antagonist mostly used in the treatment of pulmonary arterial hypertension. Ambrisentan is BCS class II drug, which suggest that it has low aqueous solubility and high membrane permeability. Ambrisentan is practically insoluble in water and in aqueous solutions at low $\mathrm{pH}[8,9]$. Because of its very poor aqueous solubility, the absorption and bioavailability of ambrisentan is incomplete. There is very few literature available regarding solubility and bioavailability improvement of ambrisentan. So in order to improve the aqueous solubility and bioavailability of the drug, an attempt was made in this present study to increase solubility, dissolution rate and bioavailability of ambrisentan by developing its solid dispersion using carrier Gelucire 50/13.

\section{MATERIALS AND METHODS}

\section{Marerials}

Ambrisentan was obtained as a gift sample from Cadila Pharmaceuticals, Mumbai India. Gelucire 50/13 was supplied by Gattefosse India as a gift sample. All other chemical and reagents used were of analytical grade.

\section{Method}

\section{Saturation solubility study of drug}

Saturation solubility study of selected drug was determined in distilled water, acetate buffer $\mathrm{pH} 1.2$, phosphate buffer $\mathrm{pH} 6.8$ and phosphate buffer pH 7.4. Extra amount of drug was added to $10 \mathrm{ml}$ study fluid in a glass vial. Samples were shaken on rotary shaker at constant speed at $25{ }^{\circ} \mathrm{C} \pm 2{ }^{\circ} \mathrm{C}$ for $48 \mathrm{~h}$. The resultant saturated solutions was then filtered using whatman filter paper no 1. Filtrates sample ware then estimated spectrophotometrically after suitable dilution [10].

\section{Phase solubility study}

In order to predict the effect carrier on the solubilization of drug, the phase solubility study of drug was carried out. Excess amount of drug were added to $10 \mathrm{ml}$ glass vial containing $0.25 \%, 0.50 \%$, $0.75 \%, 1 \%$ and $2 \%$ aqueous solution of carriers and shaken on rotary shaker for $48 \mathrm{~h}$ at a controlled temperature at $25^{\circ} \mathrm{C} \pm 2{ }^{\circ} \mathrm{C}$. The solutions were filtered using no 1 whatman filter paper. Filtrate were analyzed by UV-spectrophotometer in order to determine the concentration of the dissolved drug [11].

\section{Preparation of physical mixture}

A physical mixture of Ambrisentan with Gelucire 50/13 in different ratio $(1: 1,1: 2,1: 3,1: 4,1: 5)$ and denoted as APM 1 to APM 5 
respectively was prepared by mixing of drug and carrier using mortar and pestle. This mixture was then passed through sieve no 40 and store in desiccators. The composition was shown in table 1.

\section{Preparation of solid dispersion}

Solid dispersion of ambrisentan with Gelucire 50/13 in different weight ratio $(1: 1,1: 2,1: 3,1: 4,1: 5$ and denoted as ASD 1 to ASD 5 respectively, was prepared by kneading method. A mixture of drug and carrier was placed in a mortar and was kneaded thoroughly with water and methanol (1:1) for $20 \mathrm{~min}$. The kneaded mixtures were then dried in an oven at $40{ }^{\circ} \mathrm{C}$ until it reached the uniform weight and then pulverized and screened through 80-mesh and stored in desiccator for further study $[12,13]$. The composition for solid dispersion is shown in table 1.

Table 1: Composition of Ambrisentan solid dispersion

\begin{tabular}{lll}
\hline Formulation & $\begin{array}{l}\text { Formulation } \\
\text { code }\end{array}$ & $\begin{array}{l}\text { Ambrisentan: gelucire } \\
\mathbf{5 0 / 1 3}\end{array}$ \\
\hline Physical Mixture & APM1 & $1: 1$ \\
& APM2 & $1: 2$ \\
& APM3 & $1: 3$ \\
& APM4 & $1: 4$ \\
Solid Dispersion & APM5 & $1: 5$ \\
& ASD1 & $1: 1$ \\
& ASD2 & $1: 2$ \\
& ASD3 & $1: 3$ \\
& ASD4 & $1: 4$ \\
& ASD5 & $1: 5$ \\
\hline
\end{tabular}

\section{Characterization of solid dispersion}

\section{Determination of saturation solubility of PMs and SDs}

The saturation solubility of physical mixture and solid dispersion was determined in distilled water using shake flask method. Excess quantities of sample were added in $25 \mathrm{ml}$ of distilled water and phosphate buffer in conical flask and shaken for $24 \mathrm{~h}$ at room temperature on rotary flask shaker. After shaking resultant samples containing undissolved solid suspended in the test medium were centrifuged at 10,000 rpm for $5 \mathrm{~min}$, the clear supernatants obtained were filtered through whatman filter paper. Filtered sample ware analyzed by spectrophotometer at $263.5 \mathrm{~nm}$ after dilution [14].

\section{Determination of percent yield of solid dispersion}

The percent yield of ambrisentan solid dispersions was determined by using the following formula:

$$
\% \text { Yield }=\frac{\text { Weight of Prepared Solid Dispersion }}{\text { Weight of drug }+ \text { carrier }} \times 100
$$

\section{Determination of drug content}

Ambrisentan solid dispersion equivalent to $10 \mathrm{mg}$ of drug was accurately weighed and dissolved in methanol $(100 \mathrm{ml})$. The solution was filtered after vigorous shaken. The drug content was analyzed at $263.5 \mathrm{~nm}$ against blank by UV spectrometer after appropriate dilution [15].

\section{Fourier transform infra-red spectroscopy}

Compatibility studies of ambrisentan with carrier were performed using FTIR spectroscopy (Shimadzu FTIR-8700). Spectrum of pure drug, physical mixture and solid dispersion was recorded over the frequency range of 400 to $2000 \mathrm{~cm}-1$ at $4 \mathrm{~cm}$ resolution.

\section{Differential scanning calorimetry}

The thermal analysis was carried out using Shimadzu Thermal analyzer DT 40 (Japan). The samples were placed in sealed aluminum pans and heated at a rate of $10^{\circ} \mathrm{C}$ per min in the temperature range of $20-300^{\circ} \mathrm{C}$ under a nitrogen flow rate of $40 \mathrm{ml} / \mathrm{min}$ [16].

\section{Powder X-ray diffraction}

X-ray powder diffraction patterns of drug, carrier and solid dispersion was recorded on an X-ray powder diffraction system
(Rigaku, Mini Flex 600). The scanning was done over range of $5^{\circ}$ to $60^{\circ}$. The position and intensities of diffraction peaks were considered for the comparison of crystallinity [17].

\section{Scanning electron microscope analysis (SEM)}

The surface morphology of pure ambrisentan and selected solid dispersion was studied using SEM. (ZEISS, EVO 18, Germany). The samples were mounted on a sample stub with double-sided adhesive tape and coated under vacuum with gold ion using sputtering device prior to study. SEM image at different magnifications were recorded to study the morphological and surface characteristics of the solid dispersions.

\section{In vitro dissolution study}

In vitro dissolution study of pure ambrisentan and solid dispersions were determined using USP dissolution test apparatus II (Paddle type) (Esico International, Mumbai). Accurately weighted preparation equivalent to $10 \mathrm{mg}$ of ambrisentan ware added to 900 $\mathrm{ml}$ of phosphate buffer $\mathrm{pH} 6.8$ used as a medium of dissolution, which was maintained at $37 \pm 0.5^{\circ} \mathrm{C}$ and rotation speed was selected at $50 \mathrm{rpm} .5 \mathrm{ml}$ samples were withdrawn at time interval of 10,20 , $30,40,50,60 \mathrm{~min}$ and the same volume was replaced with fresh media in order to maintain the sink condition. After suitable dilution, collected samples were analyzed at $263.5 \mathrm{~nm}$ using UV-visible spectrophotometer against the blank $[18,19]$.

\section{In vivo/Bioavailability study}

Based on the solubility study and in vitro dissolution profile, an optimized ambrisentan SD formulation (ASD4) was selected for comparison of in vivo performance against plain ambrisentan. The pharmacokinetic study was carried out on wister rats as per the guidelines of the Committee for the Purpose of Control and Supervision on Experiments on Animals (CPCSEA), after approval of study, registration No. IAEC/2019-20/RP-09 dated 03/03/2021 at Oriental College of Pharmacy, Indore, (M. P.), India.

\section{Study design}

Healthy male wistar rats obtained from the animal house of Oriental college of Pharmacy, Indore, of weight (250-300 g) were used for the study. The rats were randomly divided into 2 groups containing 6 rats in each group. All rats were fasted overnight with free access to water prior to the experiment. The oral bioavailability of ambrisentan was determined at a dose of $10 \mathrm{mg} / \mathrm{kg}$ of body weight. The drug sample was prepared by suspending the drug in $1 \mathrm{ml}$ of $1 \% \mathrm{w} / \mathrm{v}$ aqueous sodium carboxyl methylcellulose and further diluted with water to the concentration of $1 \mathrm{mg} / \mathrm{ml}$. Based on the body weight, aqueous solution of pure ambrisentan was given orally to one group of animal, which was treated as control using oral feeding sonde. The second group of animal was administered with optimized formulation (ASD4) at the same dose and treated as test. $0.5 \mathrm{ml}$ of blood sample were withdrawn from postorbital vein sinus into micro centrifuge tube treated with EDTA at time interval of 1 , $1.5,2,2.5,3,4,6,8,12 \mathrm{hr}$. The plasma was separated by centrifugation at $3000 \mathrm{rpm}$ for $10 \mathrm{~min}$ and was treated with small quantity of acetonitrile and repeated for centrifugation for $10 \mathrm{~min}$ at $3000 \mathrm{rpm}$. The $50 \mu \mathrm{l}$ of plasma was mixed with $0.5 \mathrm{ml}$ of mobile phase. The $20 \mu \mathrm{l}$ of sample was injected with flow rate of $0.7 \mathrm{ml} / \mathrm{min}$ to C 18 column and analyzed at the wavelength of $263.5 \mathrm{~nm}$. Noncompartment pharmacokinetic parameters such as $\mathrm{T}_{\max }, \mathrm{C}_{\max }$ and AUC were estimated by PK Solver computer program. Results of in vivo experiments are reported as mean $\pm S D$ Statistical tests of significance were performed using Graph Pad Prism 5.0 software. The variables were compared with a one-way ANOVA. P-value less than 0.05 were considered significant $[20,21]$.

\section{Ex vivo permeation study}

Ex vivo permeation study was performed on pure drug and optimized SD formulation (ASD4) using an everted chicken intestine model. The study was performed using modified apparatus and USP type II dissolution test apparatus according to the method reported by Tekade et al. [22]. A freshly procured chicken intestine from local slaughterhouse was used for the study. The selected intestinal 
portion was rinsed with phosphate buffer solution (pH 6.8, KrebsRinger solution). A $6 \mathrm{~cm}$ segment of the intestine was taken and everted using glass rod and then clamped to arm B of modified apparatus. The total volume of absorption compartment was $55 \mathrm{ml}$. The apparatus was then placed in dissolution apparatus containing $1000 \mathrm{ml}$ of phosphate buffer solution $(\mathrm{pH}$ 6.8) as a dissolution medium at $37 \pm 0.5^{\circ} \mathrm{C}$. The pure drug and optimized solid dispersion was transferred to the dissolution medium and apparatus was rotated at a speed of $75 \mathrm{rpm}$. The amount of drug diffused from dissolution medium (mucosal side) to the absorption compartment side was measured by withdrawing $5 \mathrm{ml}$ sample from absorption compartment (arm B) at 5, 10, 20, 30, 40, 50 and $60 \mathrm{~min}$ and analyzed spectrophotometrically at $263.5 \mathrm{~nm}$. The experiment was carried out in triplicate $(\mathrm{n}=3$ )

\section{RESULTS AND DISCUSSION}

\section{Saturation solubility study of drug}

Saturation solubility study indicates that ambrisentan was poorly soluble in water, showing $7.347 \pm 0.003 \mu \mathrm{g} / \mathrm{ml}$ of solubility in distilled water. Ambrisentan shown $\mathrm{pH}$ dependent solubility, solubility of ambrisentan increases as the $\mathrm{pH}$ of solvent increases. Solubility of drug in acetate buffer $\mathrm{pH} 1.2$, phosphate buffer $\mathrm{pH} 6.8$ and $\mathrm{pH} 7.4$ was found to be $5.173 \pm 0.006 \mu \mathrm{g} / \mathrm{ml}, 28.507 \pm 0.012 \mu \mathrm{g} / \mathrm{ml}$ and $35.391 \pm 0.004 \mu \mathrm{g} / \mathrm{ml}$, respectively. Solubility profile of the drug is shown in fig. 1.

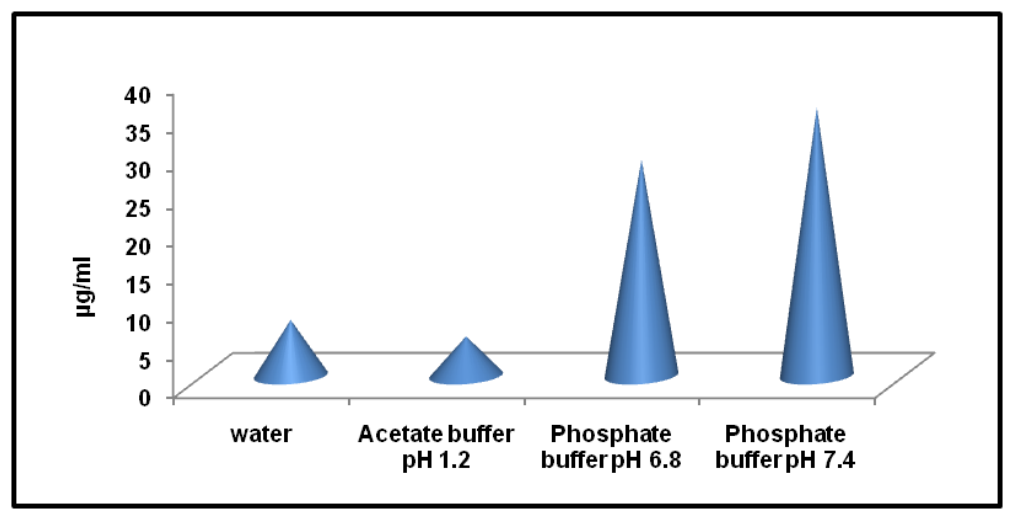

Fig. 1: Solubility profile of ambrisentan in different solvent

\section{Phase solubility study}

In order to determine the possible solubilizing effect of Gelucire 50/13 on drug solubility, phase solubility study of ambrisentan was studied using an increasing concentration of carrier. A linear increase in the solubility of drug was seen with an increasing concentration of hydrophilic carriers in water. The solubility of ambrisentan at 0.25 , $0.5,0.75,1$ and $2 \%$ aqueous solution of Gelucire $50 / 13$ was found to be $16.89 \pm 2.13, \quad 21.30 \pm 1.26, \quad 27.18 \pm 0.94, \quad 32.32 \pm 3.21, \quad 48.49 \pm 1.86$ $\mu \mathrm{g} / \mathrm{ml}$, respectively. Increased solubility may be due solubilization effect of Gelucire 50/13 that increased the wettability of the drug. At $2 \% \mathrm{w} / \mathrm{v}$ concentration of carrier, the aqueous solubility of ambrisentan was increased by 6.6 fold, indicating good affinity between drug and polymer. The phase-solubility diagram investigated for Gelucire 50/13 in distilled water was linear giving $A_{L}$ type solubility curve. The apparent stability constant $(\mathrm{Kc})$ calculated from the linear plot of the phase solubility diagram, was found to be 143.60 $\mathrm{M}^{-1}$. The Kc values were falls in the ideal range of (50 to $\left.2000 \mathrm{M}^{-1}\right)$ indicate stronger interactions between the drug and carrier [23]. The phase solubility curve of ambrisentan is shown in fig. 2 .

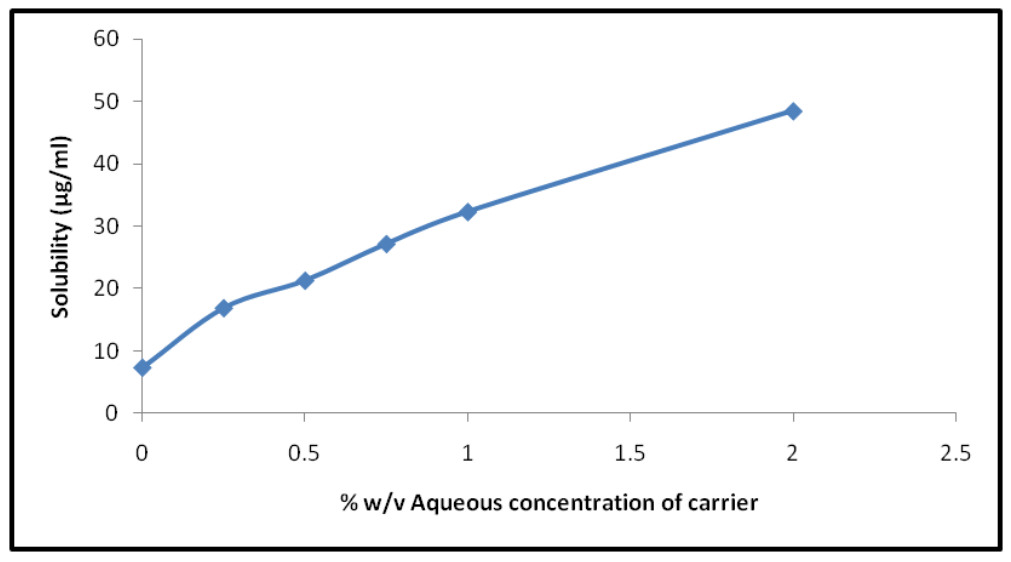

Fig. 2: Phase solubility study of ambrisentan with gelucire 50/13

\section{Saturation solubility study of solid dispersion and physical mixture}

The solubility of ambrisentan physical mixture and solid dispersion was determined in distilled water and phosphate buffer 6.8. Prepared physical mixture showed improved solubility as compare to pure drug in both solvent. Solubility study of solid dispersion showed multi-fold increase in solubility of the drug when compare with pure and physical mixture of drug. It was observed that solubility of the drug increases with increase in carrier concentration up to 1:4 ratio, but after that no significant increase in drug solubility was observed by increasing the carrier ratio. Solid dispersion ASD4 (1:4 ratio) showed maximum solubility of the drug, giving 8.23 fold increase in water solubility of ambrisentan. Higher 
value of solubility was shown by all SD formulations, this may be due to conversion of drug in amorphous form or by the increased wet ability of drug by hydrophilic carrier [24]. All SD formulations showed higher solubility of the drug in phosphate buffer solution than distilled water. The solubility data of all the PMs and SDs formulations are presented in table 2 .

Table 2: Solubility analysis of ambrisentan-gelucire 50/13 physical mixture and solid dispersion

\begin{tabular}{lllll}
\hline $\begin{array}{l}\text { Formulation } \\
\text { code }\end{array}$ & $\begin{array}{l}\text { Distilled water } \\
(\boldsymbol{\mu} \mathbf{g} / \mathbf{m l})\end{array}$ & $\begin{array}{l}\text { Phosphate buffer } \mathbf{p H} \text { 6.8 } \\
(\boldsymbol{\mu} \mathbf{g} / \mathbf{m l})\end{array}$ & $\begin{array}{l}\text { Formulation } \\
\text { code }\end{array}$ & $\begin{array}{l}\text { Distilled water } \\
(\boldsymbol{\mu} \mathbf{g} / \mathbf{m l})\end{array}$ \\
\hline APM1 & $19.41 \pm 1.30$ & $91.43 \pm 1.16$ & ASD1 & $32.14 \pm 0.94$ \\
APM2 & $22.12 \pm 1.41$ & $108.86 \pm 0.46$ & ASD2 & $40.25 \pm 0.47$ \\
APM3 & $25.61 \pm 0.84$ & $124.42 \pm 0.62$ & ASD3 & $48.74 \pm 0.81$ \\
APM4 & $28.58 \pm 1.14$ & $140.32 \pm 1.21$ & ASD & $60.46 \pm 1.63$ \\
APM5 & $31.41 \pm 0.81$ & $154.52 \pm 1.63$ & ASD5 & $62.24 \pm 1.24$ \\
\hline
\end{tabular}

Data given in mean $\pm \mathrm{SD}, \mathrm{n}=3$

\section{Fourier transform infrared spectroscopy}

IR spectra of ambrisentan and its SD with Gelucire 50/13 are presented in fig. 2. Sharp characteristic peaks showed in pure ambrisentan ware also appears in the spectra of SD, indicating no interaction between the drug and the carrier (Gelucire 50/13). FTIR spectra of pure ambrisentan, Gelucire 50/13 and SD are shown in fig. 3.

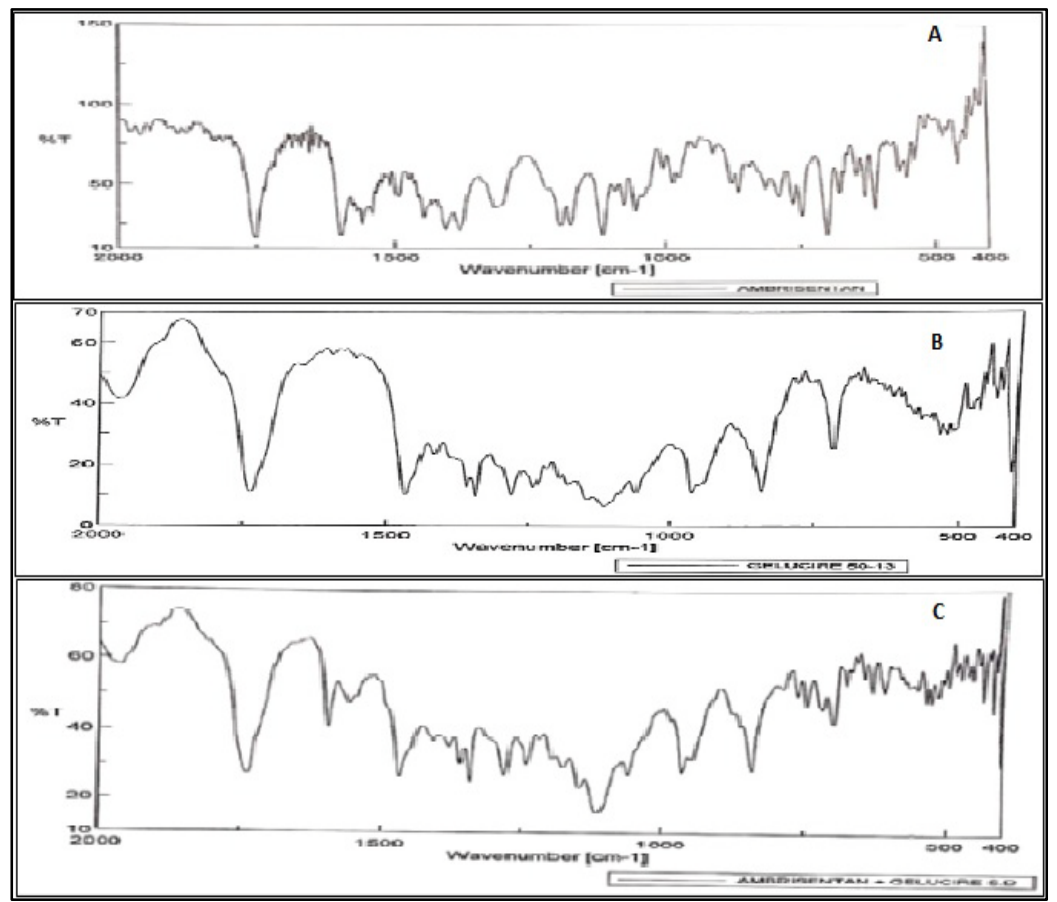

Fig. 3: FTIR Spectra (A) Ambrisentan (B) Gelucire 50/13 (C) Ambrientan Gelucire SD

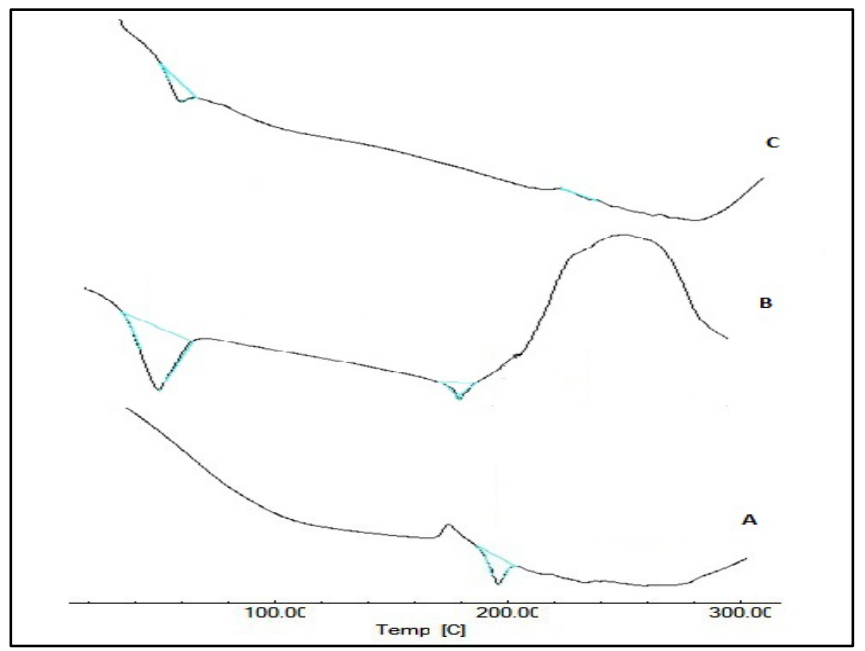

Fig. 4: DSC thermogram of (A) Pure ambrisentan, (B) Gelucire 50/13 and (C) ambrisenatn Gelucire SD 


\section{Differential scanning calorimetry}

The DSC thermogram of pure ambrisentan, Gelucire 50/13 and SD formulation (ASD4) were presented in fig. 4. Pure ambrisentan showed a single sharp endothermic peak at $194.16{ }^{\circ} \mathrm{C}$ with an enthalpy of fusion $(\Delta \mathrm{H})-10.20 \mathrm{~J} / \mathrm{g}$ corresponding to its melting point, indicates its crystalline nature. Gelucire 50/13 had showed main sharp endothermic peak at $56.78{ }^{\circ} \mathrm{C}$ with enthalpy of fusion $(\Delta \mathrm{H})$ $72.76 \mathrm{~J} / \mathrm{g}$ and another short endothermic peak at $185.78{ }^{\circ} \mathrm{C}$. The optimized solid dispersion formulation (ASD4) showed only single less intense endothermic peak at $51.37^{\circ} \mathrm{C}$, corresponding to peak of Gelucire 50/13 and does not showed an characteristic endothermic peak of ambrisentan, which indicates the complete conversion of crystalline form of drug in to amorphous form, [25] which results in enhanced solubility and dissolution rate of drug.

\section{Powder X-ray diffraction}

XRD patterns of ambrisentan, Gelucire 50/13 and ambrisentan: Gelucire 50/13 SD are shown in fig. 5. The x-ray diffractograms of pure ambrisentan showed characteristic sharp high-intensity diffraction peaks at $2 \theta$ values of $12.26^{\circ}, 14.09^{\circ}, 18.21^{\circ}, 20.48^{\circ}$, $22.77^{\circ}$ and $24.16^{\circ}$ indicates the highly crystalline nature of drug. XRD pattern of Gelucire 50/13 showed sharp high-intensity peak at a diffraction angle at $2 \theta$ value of $19.30^{\circ}$ and $23.35^{\circ}$. XRD diffractograms of solid dispersion (ASD4) showed only two prominent sharp peak at $2 \theta$ values of $19.02^{\circ}$ and $23.11^{\circ}$, corresponding to peak of Gelucire 50/13, with marked reduction in intensity or almost absence of the numerous distinctive peaks of ambrisentan. This indicates complete conversion of crystalline drug in to amorphous form in solid dispersion [26, 27].

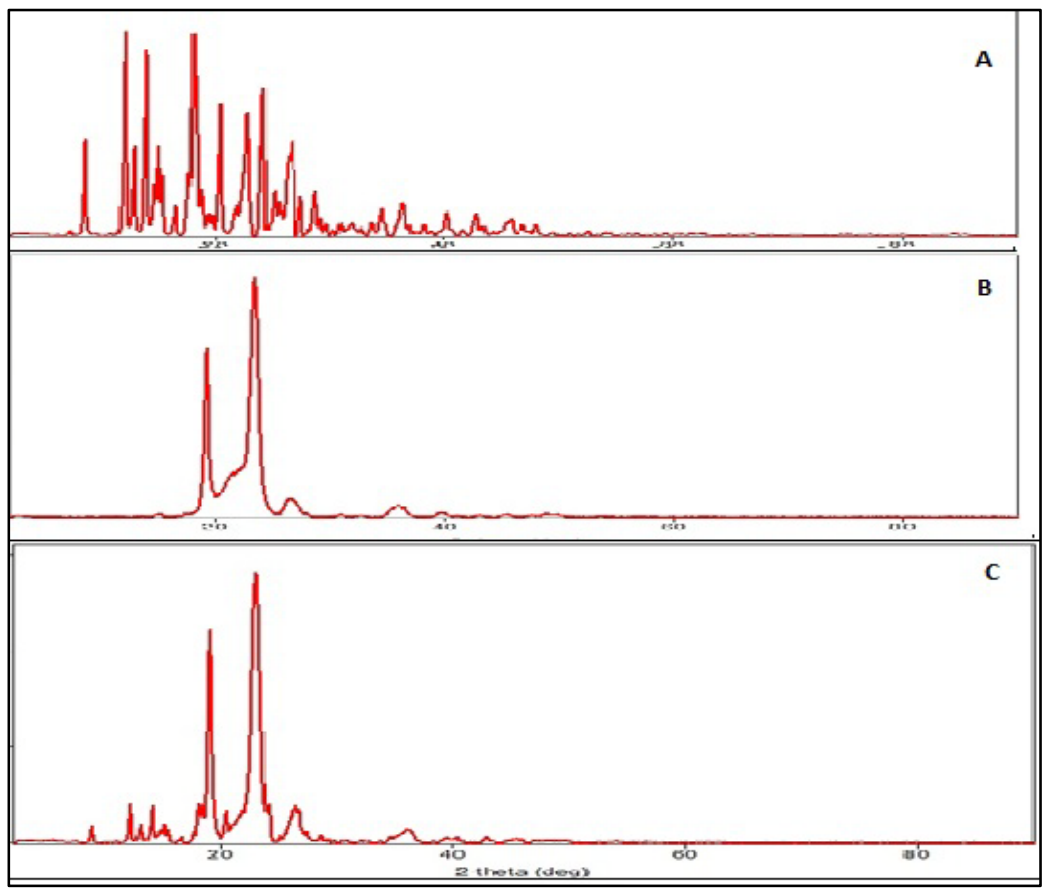

Fig. 5: XRD spectra (A) Ambrisentan, (B) Gelucire 50/13, (C) Ambrisentan Gelucire SD
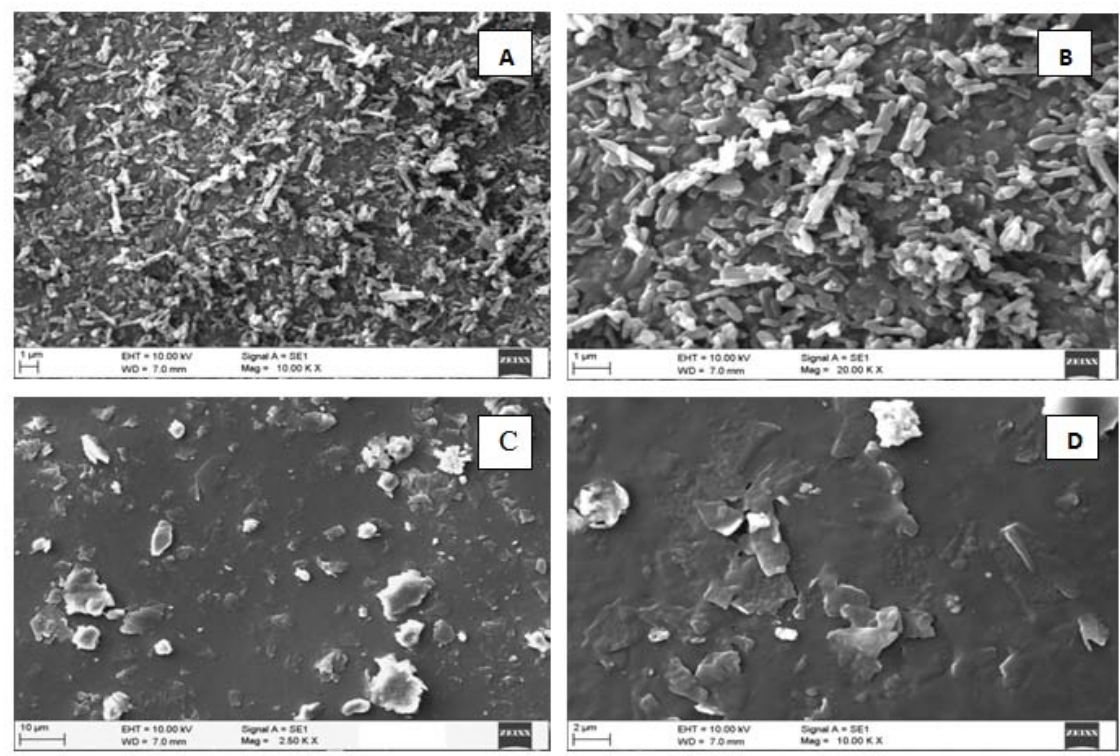

Fig. 6: SEM image of pure ambrisentan (A and B) and selected ambrisentan gelucire SD (ASD4) (C and D) at different magnification 


\section{Scanning electron microscopy (SEM)}

Scanning electron micrograph of pure ambrisentan shows rod shaped crystals indicating the crystalline nature of the drug (fig. 6A and 6B). The SEM images of selected solid dispersions (ASD4) clearly showed disappearance of rod shape crystals and formation of homogeneous smooth surface indicating complete entrapment, encapsulation or solubilization of drug on a molecular level into the polymeric matrix which suggest the conversion of drug in amorphous form (fig. 6C and 6D) [25]. This reduced crystallinity of ambrisentan in solid dispersion was further supported by the results of XRD and DSC studies.

\section{In vitro dissolution study}

All the SD formulations and the pure ambrisentan were subjected to a dissolution study. Phosphate buffers $\mathrm{pH} 6.8$ was used as the dissolution media to performed the study. Percent drug release of pure ambrisentan showed $41.15 \%$ in $60 \mathrm{~min}$, indicating its poor solubility and dissolution rate. SD formulation ASD1 to ASD5 prepared with Gelucire 50/13 showed an improved dissolution rate of $91.18 \%, 94.95 \%, 97.58 \% .101 .5 \%$ and $98.88 \%$ drug release respectively in $60 \mathrm{~min}$. It was observed that rate of drug dissolution increases with an increased in carrier concentration.

All SD formulations showed improved and faster drug dissolution rate as compare to pure drug; this may be due to combined effect of dispersibility, improved wettability and reduction of particle size in kneading technique [28]. All SD formulations showed more than $50 \%$ drug release in 10 min showing its significant improvement in drug dissolution. Among all SD formulations, ASD4 containing drug and Gelucire 50/13 in 1:4 drug to polymer ratio showed highest drug release of $101.5 \%$. The drug release profile of all SD formulations and pure ambrisentan are shown in fig. 7.

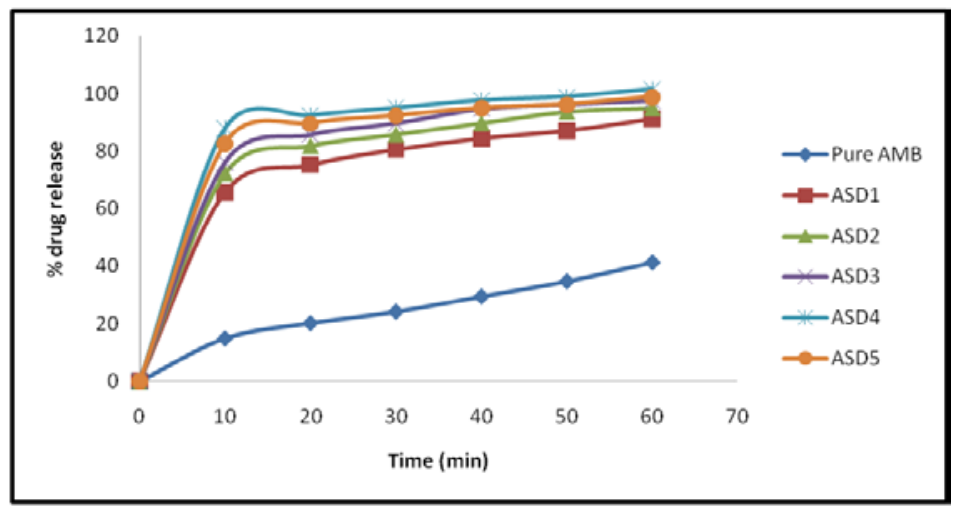

Fig. 7: In vitro dissolution profile of pure ambrisentan and ambrisentan gelucire 50/13 SDs in $6.8 \mathrm{pH}$ phosphate buffer

Table 3: \% Drug content and practical yield of SD formulations (ASD1 to ASD5)

\begin{tabular}{llll}
\hline S. No. & Formulation code & \% Practical yield & \% Drug content \\
\hline 1 & ASD1 & 80.43 & $98.15 \pm 1.39$ \\
2 & ASD2 & 81.27 & $97.26 \pm 0.38$ \\
3 & ASD3 & 81.61 & $97.56 \pm 0.26$ \\
4 & ASD4 & 83.58 & $98.62 \pm 1.18$ \\
5 & ASD5 & 83.54 & $98.12 \pm 0.24$ \\
\hline
\end{tabular}

*Data given in mean $\pm \mathrm{SD}, \mathrm{n}=3$

\section{Drug content and percentage practical yield}

Drug content for solid dispersion formulations ASD1 to ASD5 was found to be in the range of $97.26 \%$ to $98.62 \%$. The percentage drug content for all SDs formulation was found within pharmacopoeial limit which indicate uniform distribution of drug in solid dispersion.

The percentage practical yields calculated for all SDs formulation was shown in table 3. SDs formulation prepared with Gelucire 50/13 gives low yield, this may be due to sticky nature of Gelucire 50/13 which get adhere during formulation process.

\section{In vivo pharmacokinetic study}

Based on the solubility study and in vitro dissolution profile of drug, an optimized formulation, (ASD4) containing Ambrisentan: Gelucire 50/13 (1:4 ratio) was selected for comparison of in vivo performance against plain ambrisentan. The non-compartmental pharmacokinetic parameters of drug were determined after oral administration of pure ambrisentan (Control) and solid dispersion of ambrisentan (ASD4) (Test) to wistar rats by HPLC method. The peak plasma concentration $\left(\mathrm{C}_{\max }\right)$ of pure ambrisentan and its $\mathrm{SD}$ formulation was found as $4.12 \pm 0.021 \mu \mathrm{g} / \mathrm{ml}$ in $2 \mathrm{~h}, 8.04 \pm 0.100$ $\mu \mathrm{g} / \mathrm{ml}$ in $1.5 \mathrm{~h}$ respectively. From the peak plasma concentration data it was observed that absorption of drug in plasma from SD formulation is more and rapid as compare to pure drug, which suggest the reduction in crystallinity and amorphous conversion of ambrisentan in solid dispersion. The $\mathrm{AUC}_{0}$ - $\mathrm{t}$ for pure and $\mathrm{SD}$ formulation of drug (ASD4) was found to be $17.77 \pm 0.148$ and $45.72 \pm 0.397 \mu \mathrm{g} / \mathrm{mlh}$, while $\mathrm{AUC}_{0}-\infty$ was found to be $20.37 \pm 0.420$ and $62.94 \pm 1.75 \mu \mathrm{g} / \mathrm{mlh}$, respectively. The pharmacokinetic parameter indicated the maximum plasma drug concentration and area under the curve was achieved by ambrisentan SD formulation. Pharmacokinetic parameter clearly suggested the enhanced bioavailability of amabrisentan in solid dispersion (ASD4) as compared to pure form. Increased wettability of drug in the presence of hydrophilic carrier Gelucire 50/13 and SD preparation by kneading method, that causes reduction in particle size, can be a another reason in bioavailability enhancement of drug [20,29]. The obtained pharmacokinetic values were analyzed using one-way ANOVA and the values were found to be significant $(\mathrm{p}<0.05)$. Different pharmacokinetic parameters of pure ambrisentan and ambrisentan SD (ASD4) is shown in table 4 and plasma concentration time profile was shown in fig. 8.

\section{Ex vivo permeation study}

The ex vivo drug absorption study was perform in order to predict the dissolution and absorption rate of pure ambrisentan and its SD formulation (ASD4) through everted chicken intestine. Pure ambrisentan showed $32.52 \%$ drug absorption, while the solid dispersion formulation (ASD4) showed 71.43\% of drug absorption in $60 \mathrm{~min}$. The rate of drug absorption from solid dispersion was 
significantly higher than the pure drug. This improvement in absorption rate of drug was might be due to the presence of Gelucire
$50 / 13$, which increased wettability drug, resulting faster dissolution [30]. The result of ex vivo permeation of drug is shown in fig. 9.

Table 4: Pharmacokinetic parameters of pure ambrisentan and solid dispersion of ambrisentan after oral dose in wistar rat

\begin{tabular}{|c|c|c|}
\hline Pharmacokinetic parameter & Pure ambrisentan & Ambrisentan solid dispersion (ASD4) \\
\hline $\mathrm{T}_{\max }(\mathrm{h})$ & $2 \pm 0.31$ & $1.5 \pm 0.54$ \\
\hline $\mathrm{C}_{\max }(\mu \mathrm{g} / \mathrm{ml})$ & $4.12 \pm 0.021$ & $8.04 \pm 0.100$ \\
\hline $\mathrm{t}_{1 / 2}(\mathrm{~h})$ & $3.35 \pm 0.112$ & $6.37 \pm 0.335$ \\
\hline $\mathrm{AUC}_{0-\mathrm{t}}(\mu \mathrm{g} / \mathrm{mlh})$ & $17.77 \pm 0.148$ & $45.72 \pm 0.397$ \\
\hline $\mathrm{AUC}_{0 \text {-inf. }}(\mu \mathrm{g} / \mathrm{mlh})$ & $20.37 \pm 0.420$ & $62.94 \pm 1.75$ \\
\hline MRT (h) & $6.28 \pm 0.191$ & $9.40 \pm 0.387$ \\
\hline
\end{tabular}

mean $\pm S D(n=6)$

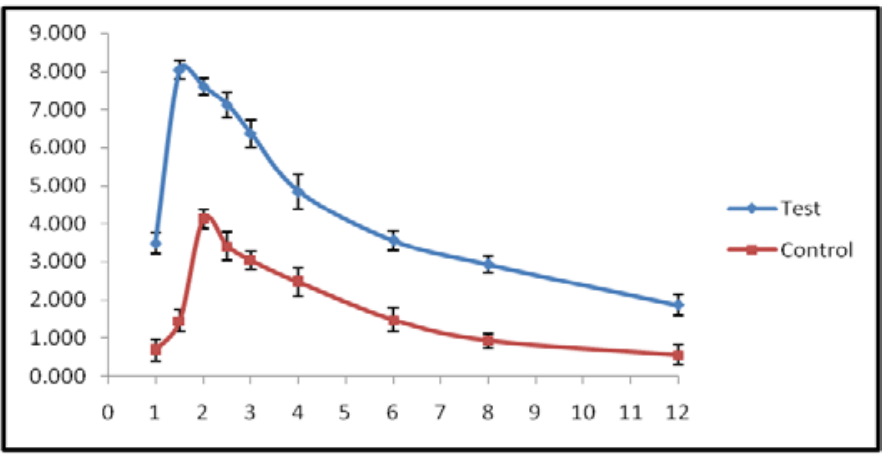

Fig. 8: Plasma concentration profile of pure ambrisentan and SD of ambrisentan (ASD4) in rats (Data are mean \pm SD (n=6))

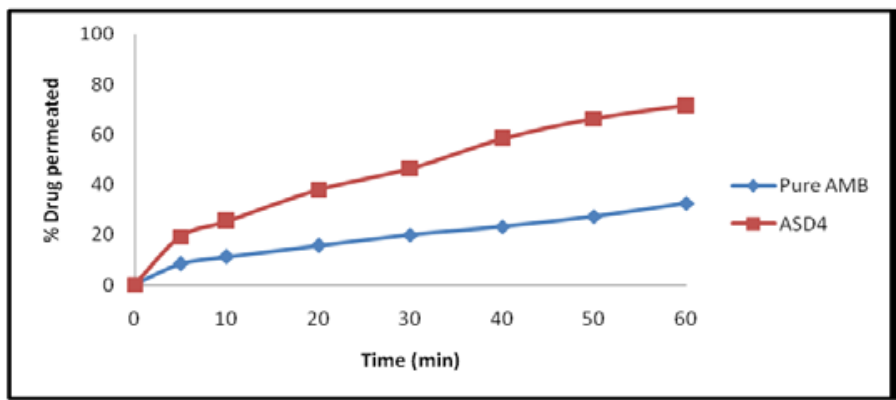

Fig. 9: Ex vivo permeation study of pure ambrisentan and its solid dispersion (ASD4)

\section{CONCLUSION}

In the present investigation, an attempt was made to develop Gelucire 50/13 based solid dispersion of poorly water-soluble ambrisentan using kneading method. The solubility and dissolution study of ambrisentan was increased multifold in solid dispersion using carrier Gelucire 50/13. DSC, XRD and SEM study confirmed the reduction in crystallinity and amorrphus conversion of drug in SD. In vivo and Ex vivo study suggests the rapid absorption and appearance of drug in plasma from SD formulation than its pure form. From this study, it was concluded that the solid dispersion prepared using Gelucire 50/13 is a good approach of solubility and bioavailability enhancement of poorly soluble drugs such as ambrisentan.

\section{ACKNOWLEDGMENT}

Authors are thankful to Cadila Pharmaceuticals for providing a gift sample of Ambrisentan to conduct this research. Also, authors are thankful to Gattefosse, India for supplying Gelucire 50/13 for completion of this research.

\section{FUNDING}

Nil

\section{AUTHORS CONTRIBUTIONS}

All authors have contributed equally.

\section{CONFLICT OF INTERESTS}

The authors declare no conflict of interest.

\section{REFERENCES}

1. Naseem A, Olliff CJ, Martini LG, Lloyd AW. Effects of plasma irradiation on the wettability and dissolution of compacts of griseofulvin. Int J Pharm. 2004;269(2):443-50. doi: 10.1016/j.ijpharm.2003.09.029, PMID 14706255.

2. Singh G, Kaur L, Gupta GD, Sharma S. Enhancement of the solubility of poorly water soluble drugs through solid dispersion: a comprehensive review. Pharm Sci. 2017;79(5):674-87. doi: 10.4172/pharmaceutical-sciences.1000279.

3. Aulton ME. Pharmaceutics: the science of dosage form design. $1^{\text {st }}$ ed. New York: Churchill Livingstone; 1996.

4. Vo CL, Park C, Lee BJ. Current trends and future perspectives of solid dispersions containing poorly water-soluble drugs. Eur J Pharm Biopharm. 2013;85(3 Pt B):799-813. doi: 10.1016/j.ejpb.2013.09.007, PMID 24056053.

5. Sareen S, Mathew G, Joseph L. Improvement in solubility of poor water-soluble drugs by solid dispersion. Int J Pharm Investig. 2012;2(1):12-7. doi: 10.4103/2230-973X.96921, PMID 23071955.

6. Raymond CR, Paul JS, Marian EQ. Handbook of pharmaceutical excipients. $6^{\text {th }}$ ed. Pharmaceutical Press; 2020. p. 557-60. 
7. Yu DG, Yang JM, Branford White CB, Lu P, Zhang L, Zhu LM. Third generation solid dispersions of ferulic acid in electrospun composite nanofibers. Int J Pharm. 2010;400(1-2):158-64. doi: 10.1016/j.ijpharm.2010.08.010, PMID 20713138.

8. European medicine agency. Evaluation of medicines for human use, Assessment report for volibris Doc. Ref.: EMEA/123999/2008; 2008. p. 1-44.

9. Barst RJ. A review of pulmonary arterial hypertension: role of ambrisentan. Vasc Health Risk Manag. 2007;3(1):11-22. PMID 17583171.

10. Higuchi T, Connors KA. Phase-solubility techniques. Adv Anal Chem Instr. 1965;4:117-212.

11. Sharma A, Jain CP. Preparation and characterization of solid dispersions of carvedilol with PVP K30. Res Pharm Sci. 2010;5(1):49-56. PMID 21589768

12. Modi A, Tayade P. Enhancement of dissolution profile by solid dispersion (Kneading) technique. AAPS PharmSciTech 2006;7(3):68. doi: 10.1208/pt070368, PMID 17025249.

13. Iqbal A, Hossain S, Shamim A, Islam M, Siddique AT. Formulation, in vitro evaluation and characterization of atorvastatin solid dispersion. Trop J Pharm Res. 2020;19(6):1131-8. doi: 10.4314/tjpr.v19i6.2.

14. Sathali AAH, Jayalakshmi J. Enhancement of solubility and dissolution rate of olmesartan medoxomil by solid dispersion technique. J Curr Chem Pharm Sci 2013;3(2):123-34.

15. Yunoos M, Lavanya NSL, Sravani G, Madhuri Rao P, Krishna CH. Development of a validated UV spectrophotometric method for the estimation of ambrisentan in pure and marketed formulations. Sch Acad J Pharmacol. 2014;3(6):427-31.

16. Yang M, Wang P, Huang CY, Ku MS, Liu H, Gogos C. Solid dispersion of acetaminophen and poly (ethylene oxide) prepared by hot-melt mixing. Int J Pharm. 2010;395(1-2):53 61. doi: 10.1016/j.ijpharm.2010.04.033, PMID 20435110.

17. Adahalli SB, Talluri M. Formulation and evaluation of tablet prepared by coamorphous system containing antihypertensive and anti-hyperlipidemic drug. Int J Pharm Pharm Sci. 2016;8(9):182-93. doi: 10.22159/ijpps.2016.v8i9.12895.

18. Varma MM, SK RB. Formulation, physicochemical evaluation and dissolution studies of carbamazepine solid dispersions. IJPSN 2012;5(3):1790-807. doi: 10.37285/ijpsn.2012.5.3.7.

19. Avachat A, Raut V. Solubility and dissolution enhancement of nebivolol hydrochloride using hydrophilic carriers. Asian J Pharm Sci. 2012;7(5):337-45

20. Deshmane S, Deshmane S, Shelke S, Biyani K. Enhancement of solubility and bioavailability of ambrisentan by solid dispersion using daucus carota as a drug carrier: formulation, characterization, in vitro, and in vivo study. Drug Dev Ind Pharm. 2018;44(6):1001-11. doi: 10.1080/03639045.2018.1428339, PMID 29382236.

21. Srinivas I, Bhikshapathi DVRN. Preparation and in vivo evaluation of solid dispersions using repaglinide. Int J Pharm Sci Drug Res. 2018;10(5):362-71.

22. Rodde MS, Divase GT, Devkar TB, Tekade AR. Solubility and bioavailability enhancement of poorly aqueous soluble atorvastatin: in vitro, ex vivo, and in vivo studies. BioMed Res Int. 2014;2014:463895. doi: 10.1155/2014/463895, PMID 24995297.

23. Maulvi FA, Dalwadi SJ, Thakkar VT, Soni TG, Gohel MC, Gandhi TR. Improvement of dissolution rate of aceclofenac by solid dispersion technique. Powder Technol. 2011;207(1-3):47-54 doi: 10.1016/j.powtec.2010.10.009.

24. Daravath B, Tadikonda RR, Vemula SK. Formulation and pharmacokinetics of gelucire solid dispersions of flurbiprofen. Drug Dev Ind Pharm. 2015;41(8):1254-62. doi: 10.3109/03639045.2014.940963, PMID 25039470.

25. Mehanna MM, Motawaa AM, Samaha MW. In: sight into tadalafil-block copolymerbinary solid dispersion: mechanistic investigation of dissolution enhancement. Int J Pharm. 2010;402(1-2):78-88.

26. Murali Mohan Babu GV, Prasad ChD, Ramana Murthy KV. Evaluation of modified gum karaya as carrier for the dissolution enhancement of poorly water-soluble drug nimodipine. Int J Pharm. 2002;234(1-2):1-17. doi: 10.1016/s0378-5173(01)00925-5, PMID 11839433

27. Deshkar S, Satpute A. Formulation and optimization of curcumin solid dispersion pellets for improved solubility. Int J Appl Pharm. 2020;12(2):36-46. doi 10.22159/ijap.2020v12i2.34846.

28. Ghareeb MM, Abdulrasool AA, Hussein AA, Noordin MI Kneading technique for preparation of solid binary dispersion of meloxicam with poloxamer 188. AAPS PharmSciTech. 2009;10(4):1206-15. doi: 10.1208/s12249-009-9316-0, PMID 19862626.

29. Liu C, Wu J, Shi B, Zhang Y, Gao T, Pei Y. Enhancing the bioavailability of cyclosporine a using solid dispersion containing polyoxyethylene (40) stearate. Drug Dev Ind Pharm. 2006;32(1):115-23. doi: 10.1080/03639040500388573, PMID 16455610 .

30. Bhalekar MR, Upadhaya PG, Reddy S, Kshirsagar SJ, Madgulkar AR. Formulation and evaluation of acyclovir nanosuspension for enhancement of oral bioavailability. Asian J Pharm. 2014;8(2):110-8. doi: 10.4103/0973-8398.134942. 